\title{
Corrigendum to "Automatic Fruit Morphology Phenome and Genetic Analysis: An Application in the Octoploid Strawberry"
}

\author{
Laura M. Zingaretti $\mathbb{D}^{1},{ }^{1}$ Amparo Monfort $\mathbb{D}^{1},{ }^{1,2}$ and Miguel Pérez-Enciso $\mathbb{D}^{1,3}$ \\ ${ }^{1}$ Centre for Research in Agricultural Genomics (CRAG), CSIC-IRTA-UAB-UB, 08193 Bellaterra, Barcelona, Spain \\ ${ }^{2}$ Institut de Recerca i Tecnologia Agroalimentàries (IRTA), 08193 Barcelona, Spain \\ ${ }^{3}$ ICREA, Passeig de Lluís Companys 23, 08010 Barcelona, Spain
}

Correspondence should be addressed to Laura M. Zingaretti; m.lau.zingaretti@gmail.com

Received 14 December 2021; Accepted 14 December 2021; Published 20 January 2022

Copyright (c) 2022 Laura M. Zingaretti et al. Exclusive Licensee Nanjing Agricultural University. Distributed under a Creative Commons Attribution License (CC BY 4.0).

In the article titled "Automatic Fruit Morphology Phenome and Genetic Analysis: An Application in the Octoploid Strawberry" [1], some funding information was omitted. The funding DOI "10.13039/501100011033" was missing. The corrected Acknowledgements section is provided below.

\section{Acknowledgments}

The authors would like to thank Planasa for providing the strawberry fruits under the Planasa-IRTA collaboration contract, headed by AM. LMZ was supported by a $\mathrm{PhD}$ grant from the Ministry of Economy and Science (MINECO, Spain). Work was funded by the MINECO grants AGL2016-78709-R and PID2019-108829RB-I00 funded by MCIN/AEI/ 10.13039/501100011033 to MPE and by the CERCA Programme/Generalitat de Catalunya. We acknowledge the financial support from the Spanish Ministry of Science and Innovation-State Research Agency (AEI), through the "Severo Ochoa Programme for Centres of Excellence in R\&D” SEV-2015-0533 and CEX2019-000902-S.

\section{References}

[1] L. Zingaretti, A. Monfort, and M. Pérez-Enciso, “Automatic Fruit Morphology Phenome and Genetic Analysis: An Application in the Octoploid Strawberry," Plant Phenomics, vol. 2021, article 9812910, pp. 1-14, 2021. 